\title{
Long non-coding RNA HOTAIR promotes tumor cell invasion and metastasis by recruiting EZH2 and repressing E-cadherin in oral squamous cell carcinoma
}

\author{
YANSHENG WU $^{1,5^{*}}$, LI ZHANG $^{3,7 *}$, LUN ZHANG $^{1,5^{*}}$, YANG WANG $^{4-6}$, \\ HUI LI ${ }^{4-6}$, XIUBAO REN ${ }^{2,4-6}$, FENG WEI ${ }^{4-6}$, WENWEN YU ${ }^{4-6}$, TING LIU ${ }^{4-6}$, \\ XUDONG WANG ${ }^{1,5}$, XUAN ZHOU ${ }^{1,5}$, JINPU YU $^{4-6}$ and XISHAN HAO ${ }^{4-6}$ \\ Departments of ${ }^{1}$ Maxillofacial and Otorhinolaryngology Head and Neck Surgery, ${ }^{2}$ Immunology
and ${ }^{3}$ Breast Cancer Medical Oncology, ${ }^{4}$ Biotherapy Center, Tianjin Medical University
Cancer Institute and Hospital; ${ }^{5}$ National Clinical Research Center of Cancer, \\ Key Laboratory of Cancer Prevention and Therapy; ${ }^{6}$ Key Laboratory of Cancer Immunology \\ and Biotherapy; ${ }^{7}$ Key Laboratory of Cancer Prevention and Therapy, Tianjin, P.R. China
}

Received February 16, 2015; Accepted March 30, 2015

DOI: 10.3892/ijo.2015.2976

\begin{abstract}
HOX transcript antisense RNA (HOTAIR), a long intergenic non-coding RNA (lncRNA), functions as a molecular scaffold to link and target the histone modification complexes PRC2 and LSD1, then reprograms chromatin states by coupling histone H3K27 methylation and H3K4 demethylation for epigenetic gene silencing to promote cancer metastasis. It is associated with poor survival in several solid cancers. In this study, we show that HOTAIR expression increased in oral squamous cell carcinoma (OSCC) compared with non-tumor tissue and is associated with metastasis, the stage and histological differentiation. In addition, overexpression of HOTAIR indicated poor overall survival (OS) and disease-free survival (DFS) in OSCC patients. Knockdown of HOTAIR by siRNA in OSCC cells decreased cell proliferation and colony formation, increased cell invasion and migration, and induced apoptosis in vitro. Furthermore, significant negative correlation between HOTAIR levels and E-cadherin levels was found in OSCC tissues and cell lines, and HOTAIR contributed to the regulation of E-cadherin through binding to EZH2 and H3K27me3 with the E-cadherin promoter. Our findings suggest that
\end{abstract}

Correspondence to: Professor Xishan Hao or Professor Jinpu Yu, Biotherapy Center, Tianjin Medical University Cancer Institute and Hospital, Tianjin, P.R. China

E-mail: hxstmu@hotmail.com

E-mail: jinpu_yu@hotmail.com

*Contributed equally

Key words: HOX transcript antisense RNA, oral squamous cell carcinoma, metastasis, EZH2, E-cadherin
HOTAIR expression is associated with OSCC and may be one of critical targets in progression and metastasis, and an indicator of poor survival in OSCC.

\section{Introduction}

Oral squamous cell carcinoma (OSCC) is one of the most common cancer affecting the head and neck region, causing $\sim 42,440$ new cases and 8,390 deaths annually in the United States. In spite of the many advances in surgical treatment, radiotherapy and chemotherapy used in OSCC, the 5-year survival rate after diagnosis for OSCC remains at $\sim 50 \%$ (1). In addition, the limited survival of OSCC patients is likely due to a high proportion of patients with advanced disease stages, lack of suitable markers for early detection, failure to respond to available drugs and incomplete knowledge of the mechanisms of the poor survival risk factors (2-5). Studies on the mechanisms underlying the malignant progression and finding a therapeutic target of OSCC might be an effective way to improve the treatment efficacy of this cancer. Recent discoveries have dramatically improved our understanding of the mechanisms of cell development and progression in OSCC. However, most studies are focusing on the proteincoding genes and the studies on non-coding RNA are limited, especially on long non-coding RNAs.

Long non-coding RNAs (lncRNAs) are transcripts longer than $200 \mathrm{nts}$, and without a functional open reading frame (ORF) in most cases, but they might play widespread roles in gene regulation and other cellular processes, including their involvement in epigenetic regulation, gene transcription, and post-transcription regulation (6). In addition, recent studies suggests that lncRNAs are involved in the development of human diseases, particularly in cancer $(7,8)$. Among the lncRNAs, HOX transcript antisense RNA (HOTAIR), a paradigm for long non-coding RNA function in cancer, is transcribed from the HOXC cluster in an antisense manner, 
functions as a molecular scaffold to link the polycomb repressive complexes 2 (PRC2) and the lysine specific demethylase 1 (LSD1) complexes, then regulates gene expression by mediating the modulation of chromatin structures in trans across the 40-kb HOXD locus. Evidence has shown that HOTAIR is pervasively overexpressed in many cancers and involved in tumor invasion, progression, metastasis and poor prognosis (9-11). However, the functions and the molecular mechanisms of HOTAIR in OSCC are still unknown. In the present studies, we measured the expression of HOTAIR and the relationship with clinicopathological factors and survival in OSCC patients. We investigated the functions of HOTAIR involvement in OSCC cell proliferation, invasion and apoptosis in vitro. Interestingly, we found that HOTAIR recruits EZH2 and represses E-cadherin in OSCC. In addition, the study results may assist to reveal the mechanism of long noncoding RNA HOTAIR in OSCC and to find the molecular targets to inhibit invasion and metastasis in OSCC.

\section{Materials and methods}

OSCC patients and cell lines. OSCC samples and adjacent histological normal tissues were obtained from 76 patients who were admitted to the Department of Maxillofacial Surgery and and Otorhinolaryngology Head and Neck Surgery of Tianjin Medical University between January 2001 and March 2009. None of the patients received treatment prior to radical surgical treatment. Tumor tissues and non-malignant tissues that were $\geq 1.5 \mathrm{~cm}$ distal to the tumor margins were snap-frozen in liquid nitrogen and then stored at $-80^{\circ} \mathrm{C}$ until use. The clinicopathological characteristics of patients are summarized in Table I. The study was approved by the Institutional Ethics Committee of Tianjin Medical University Cancer Institute and Hospital, and patients signed a statement of informed consent before entry into the study.

Two OSCC cell lines (TSCCA, Tca8223) were purchased from the Institute of Basic Medical Sciences of Chinese Academy of Medical Sciences (Beijing, China). CAL-27 cell line was purchased from Cell Resource Center, IBMS, CAMS/PUMC (Beijing, China), and Tb3.1 cell line was obtained from Shanghai Ninth People's Hospital (Shanghai, China). Cells were cultured in RPMI-1640, DMEM or MEM (Invitrogen, CA, USA) medium supplemented with $10 \%$ fetal bovine serum (FBS), (Hyclone, UT, USA) in humidified air with $5 \% \mathrm{CO}_{2}$ at $37^{\circ} \mathrm{C}$.

RNA preparation, reverse transcription, and quantitative real-time $P C R$. Total RNA was extracted from tissues or cultured cells with TRIzol reagent (Invitrogen, NY, USA) according to the manufacturer's protocol. qRT-PCR assays were performed to detect HOTAIR expression using the PrimeScript RT reagent kit and SYBR Premix Ex Taq (Takara, Dalian, China) according to the manufacturer's instructions. Results were normalized to the expression of glyceraldehyde3-phosphate dehydrogenase (GAPDH). The primers used were as follows: HOTAIR F: 5'-AAATATGGCGGCGTCTA CACGGA-3', R: 5'-TCCAGAACCCTCTGACATTTGCCT-3'; E-cadherin F: 5'-TGCCCAGAAAATGAAAAAGG-3', R: 5'-GTGTATGTGGCAATGCGTTC-3'; GAPDH F: 5'-GAA GGTGAAGGTCGGAGTC-3', R: 5'-GAAGATGGTGATGG
GATTTC-3'; EZH2 F: 5'-AATCAGAGTACATGCGACT GAGA-3', R: 5'-GCTGTATCCTTCGCTGTTTCC-3'. qRT-PCR and data collection were performed on an ABI 7500 (Applied Biosystems, CA, USA). qRT-PCR results were analyzed and expressed relative to $\mathrm{CT}$ (threshold cycle) values, and then converted to fold changes.

Transfection of siRNA. Three individual FAM-siRNAs (siHOTAIR1, siHOTAIR2 and siHOTAIR3) siSUZ12 and siEZH2, negative control FAM-siRNA (silencer negative control siRNA) were purchased from GenePharma (Shanghai, China). siRNA oligonucleotides (10 nmol/l) in Opti-MEM (Invitrogen, NY,USA) were transfected into cells using Lipofectamine 2000 (Invitrogen, NY, USA) following the manufacturer's protocol. Transfection efficiencies were detected by fluorescence microscope 6 and $48 \mathrm{~h}$ post-transfection, HOTAIR expression levels were measured. Target sequences for HOTAIR siRNAs were as follows: siHOTAIR1 sense, 5'-GCACAGAGCAACU CUAUAATT-3'; antisense, 5'-UUAUAGAGUUGCUCUGUG CTT-3'. siHOTAIR2 sense, 5'-GCCUUUGGAAGCUCUUG AATT -3'; antisense, 5'-UUCAAGAGCUUCCAAGGCTT-3'. siCT sense, 5'-UUCUCCGAACGUGUCACGUTT-3'; antisense, 5'-ACGUGACACGUUCGGAGAATT-3'. siEZH2 sense, 5'-TTCATGCAACACCCAACACT-3'; siEZH2 antisense, 5'-GAGAGCAGCAGCAAACTCCT-3'.

Wound healing scratch assay. Cells were seeded in 6-well plates in normal cell growth media and incubated to confluence. A $20-\mu 1$ tip was used to make a straight scratch, simulating a wound. The medium was changed to MEM or 1640 containing $2 \%$ FBS. After 48-h incubation, the area occupied by migrated cells in the scratch was evaluated.

Matrigel invasion assay. The Matrigel invasion assay was done using the BD Biocoat Matrigel Invasion Chamber (pore size, $8 \mathrm{~mm}, 24$-well; BD Biosciences, CA, USA) following the manufacturer's protocol. Cells were plated in the upper chamber in serum-free medium. The bottom chamber contained medium with $10 \%$ FBS. After $48 \mathrm{~h}$, the bottom of the chamber insert was stained with methanol and $0.1 \%$ crystal violet, imaged, and counted using an IX70 inverted microscope (Olympus, Tokyo, Japan). Each Matrigel invasion assay was conducted in at least 3 replicates.

Flow cytometric analysis of apoptosis. TSCCA and Tca8113 cells, transiently transfected with siHOTAIR or siCT, were harvested $48 \mathrm{~h}$ after transfection by trypsinization. After double staining with FITC-Annexin V and propidium iodide, cells were analyzed by flow cytometry using CellQuest software (BD Biosciences). Cells were discriminated into viable cells, dead cells, early apoptotic cells, and apoptotic cells, and then the relative ratio of apoptotic cells was compared to the control from each experiment. All samples were assayed in triplicate.

Colony formation assay. A total of 500 HOTAIR siRNAtransfected Tscca and Tca8113 cells were placed in a fresh 6 -well plate and maintained in media containing 10\% FBS, replacing the medium every 3 days. After 14 days, cells were fixed with methanol and stained with $0.1 \%$ crystal violet. 
Table I. HOTAIR expression and clinicopathological factors in OSCC.

\begin{tabular}{|c|c|c|c|c|}
\hline \multirow[b]{2}{*}{$\begin{array}{l}\text { Clinicopathological } \\
\text { factors }\end{array}$} & \multirow[b]{2}{*}{$\mathrm{n}(\%)$} & \multicolumn{2}{|c|}{$\begin{array}{l}\text { HOTAIR } \\
\text { expression }\end{array}$} & \multirow[b]{2}{*}{ P-value } \\
\hline & & High & Low & \\
\hline Gender & & & & 0.479 \\
\hline Male & $47(62)$ & 22 & 25 & \\
\hline Famale & $29(38)$ & 16 & 13 & \\
\hline Age (years) & & & & 0.243 \\
\hline$\leq 60$ & $45(59)$ & 20 & 25 & \\
\hline$>60$ & $31(41)$ & 18 & 13 & \\
\hline \multicolumn{4}{|l|}{ Smoking and/or } & 0.238 \\
\hline Yes & $47(62)$ & 26 & 21 & \\
\hline No & $29(38)$ & 12 & 17 & \\
\hline \multicolumn{4}{|l|}{ Location } & 0.065 \\
\hline OTSCC $^{a}$ & $42(55)$ & 25 & 17 & \\
\hline OSCC & $34(45)$ & 13 & 21 & \\
\hline \multicolumn{5}{|l|}{ OTSCC (excepted) } \\
\hline Tumor size $(\mathrm{cm})$ & & & & 0.758 \\
\hline$\leq 2$ & $21(28)$ & 11 & 10 & \\
\hline $2-4$ & $41(54)$ & 19 & 22 & \\
\hline$>4$ & $14(18)$ & 8 & 6 & \\
\hline $\begin{array}{l}\text { Histological } \\
\text { differentiation }\end{array}$ & \multicolumn{3}{|c|}{ Histological } & 0.019 \\
\hline High & $29(38)$ & 9 & 20 & \\
\hline Median & $35(46)$ & 20 & 15 & \\
\hline Poor & $12(16)$ & 9 & 3 & \\
\hline Stage & & & & 0.001 \\
\hline I-II & $32(42)$ & 9 & 23 & \\
\hline III-IV & $44(58)$ & 29 & 15 & \\
\hline \multicolumn{5}{|l|}{ Lymph node } \\
\hline metastasis & & & & 0.000 \\
\hline $\mathrm{N} 1$ & $34(45)$ & 26 & 8 & \\
\hline NO & $42(55)$ & 12 & 30 & \\
\hline
\end{tabular}

${ }^{\mathrm{a} O T S C C}$, oral tongue squamous cell carcinoma.

Visible colonies were manually counted. For each treatment group wells were measured in triplicate.

Western blot analysis and antibodies. Cells were lysed using RIPA protein extraction reagent (Beyotime, Shanghai, China) supplemented with a protease inhibitor cocktail (Roche, Basel, Switzerland). Protein concentration was measured using the Bio-Rad protein assay kit (Bio-Rad, Beijing, China). Approximately $30 \mu \mathrm{g}$ of protein extract was separated by $10 \%$ SDS polyacrylamide gel electrophoresis, then transferred to nitrocellulose membrane (Sigma, MO, USA) and incubated with mouse anti-human E-cadherin, EZH2, H3K27me3 (Abcam, MA, USA). ECL chromogenic substrate was used to visualize the bands and the density of the band on the western blotting was measured using the ChemiDoc XRS imaging system and QuantityOne software (Bio-Rad). $\beta$-actin was used as a control.

Chromatin immunoprecipitation (ChIP) assay. ChIP assay was performed with the EZ-Magna ChIP kit (Millipore) according to the manufacturer's instructions. For each ChIP assay, $2 \mu \mathrm{g}$ of antibodies were used: EZH2 (Cell Signaling Technology), H3K27me3 (Abcam) and H3 (Cell Signaling Technology). The percentage of the bound DNA was quantified against the original DNA input by qRT-PCR analysis. Primer sequences used are as follows: E-cadherin-a sense, 5'-TGTCCGCCCCG ACTTGTCTCTC-3'; antisense, 5'-GTCCTCTGGCCCCAGC CTCTCT-3'.E-cadherin-b sense, 5'-AGACCCCATCTCCAAA ACGAACAAA-3'; antisense, 5'-GCATAGACGCGGTGACCC TCTAGCC-3'. E-cadherin-c sense, 5'-TGTCCGCCCCGACT TGTCTCTC-3'; antisense, CGGTCCTCTGGCCCCAGCC TCT-3'.

Statistical analysis. Statistical tests for data analysis included the Wilcoxon test, $\chi^{2}$ test, Fisher's exact test and Student's two-tailed t-test. Kaplan-Meier analysis and log-rank test were applied to evaluate the prognostic significance of HOTAIR expression level in terms of patient survival. The statistical analyses were performed using SPSS 19.0 statistical software package (SPSS, Chicago, IL, USA). The data represent the mean $\pm \mathrm{SD}$. P-values of $\mathrm{P}<0.05$ were considered to be statistically significant.

\section{Results}

HOTAIR is significantly upregulated in human OSCC tissues. Expression level of HOTAIR was detected in 76 OSCC samples and adjacent normal tissues by qRT-PCR and normalized to GAPDH. HOTAIR was more highly expressed in OSCC tumor compared with non-tumor tissues $(\mathrm{P}<0.001)$ (Fig. 1A), and HOTAIR was more highly expressed in tumors with regional lymph node metastasis (N1) compared with tumors without lymph node metastasis (N0) $(\mathrm{P}<0.001)$ (Fig. 1B). The univariate analysis revealed that HOTAIR expression strongly correlated with the clinical stage, lymph node metastasis, and histological differentiation in patients with OSCC (Table I). Kaplan-Meier survival analysis showed that patients with high HOTAIR expression had significantly decreased disease-free survival (DFS) (Fig. 1C) and overall survival (OS) (Fig. 1D) compared with patients with low HOTAIR expression. These results suggest that the HOTAIR is overexpressed in OSCC and is associated with development and progression of OSCC patients. Moreover, HOTAIR may be a potential predictive biomarker for disease outcome and prognosis in OSCC patients.

HOTAIR promotes the malignancy of OSCC cells in vivo. Next, we performed qRT-PCR analysis to examine the expression level of HOTAIR in human OSCC cell lines. Of these 4 OSCC cell lines, TSCCA and Tca8113 expressed signifi- 
A

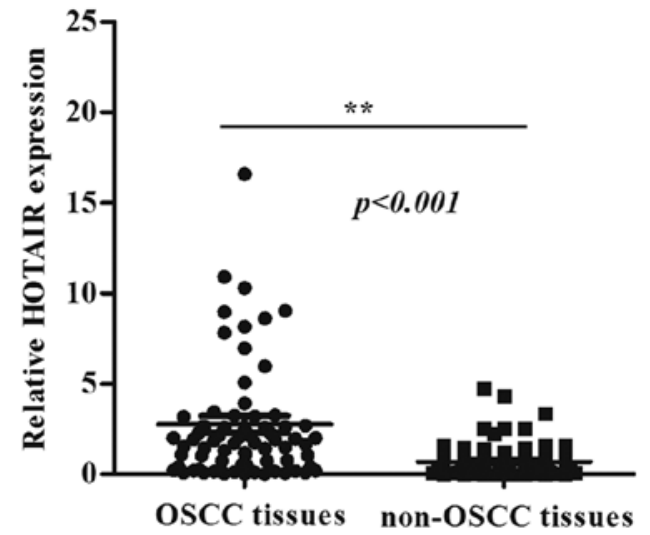

C

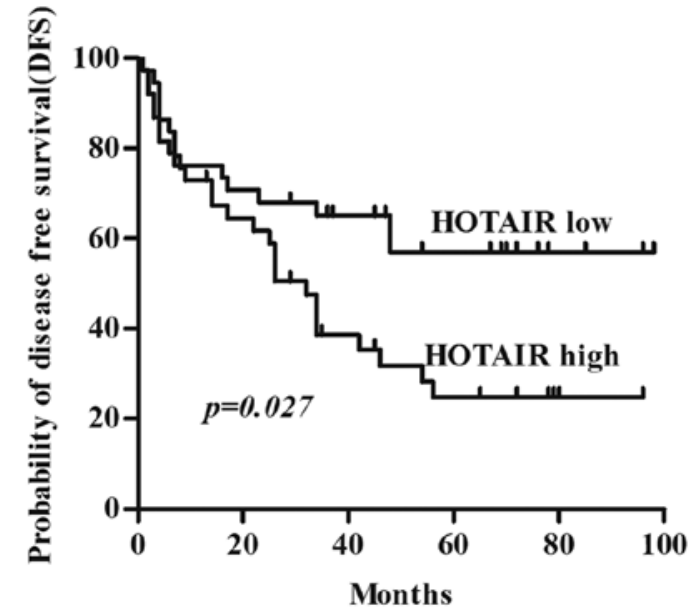

B

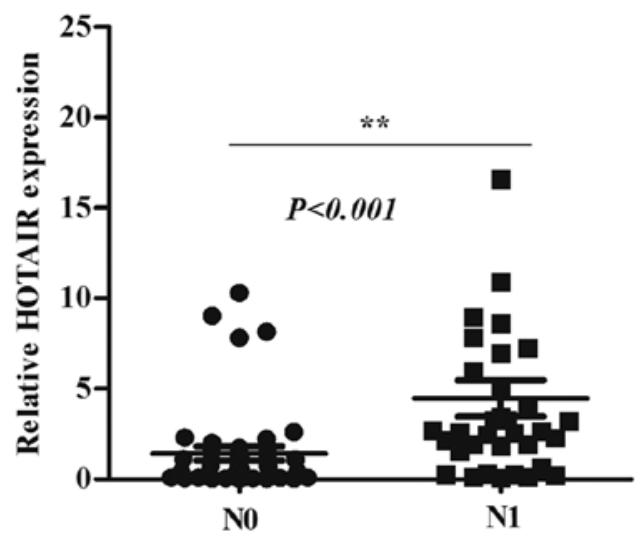

D

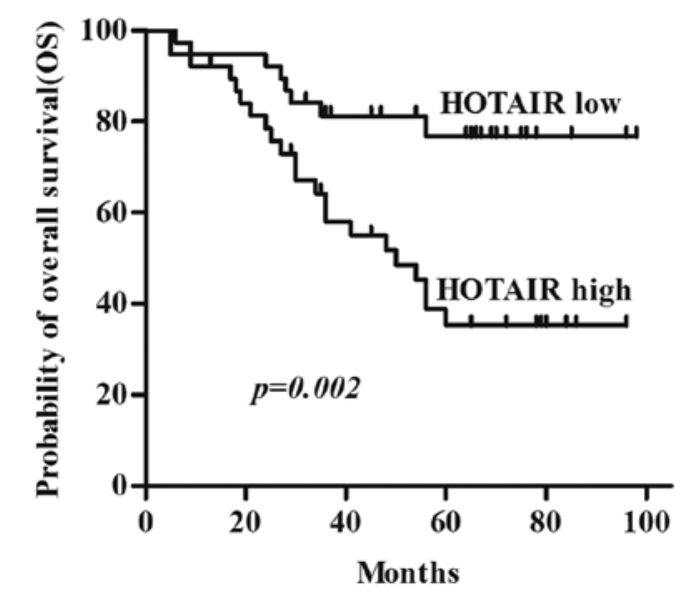

Figure 1. HOTAIR expression and prognostic significance in OSCC cancer. (A) HOTAIR expression in non-tumor and OSCC tissues from patients. (B) Relative expression of HOTAIR in OSCC patients with tumor localized in the oral cavity (N0) or those with tumors spread to cervical lymph nodes (N1). (C and D) Kaplan-Meier plot for disease-free survival (C) and overall survival (D) of OSCC patients expressing high and low levels of HOTAIR. "P<0.05.

cantly higher levels of HOTAIR (Fig. 2A). In TSCCA and Tca8113 cells, HOTAIR knockdown was performed using two different siRNAs (siHOTAIR1\# and siHOTAIR2\#), and siCT was performed as control. Lipofectamine 2000 transfection reagent was used for siRNA transfection. Forty-eight hours after treatment, HOTAIR expression was effectively knocked down in TSCCA and Tca8113 cells (Fig. 2B).

Given that overexpression of HOTAIR was significantly associated with progression in patients with OSCC we examined whether HOTAIR regulated the proliferation of OSCC cells. MTT assay was used to examine the effect of HOTAIR silence on the proliferation of TSCCA and Tca8113 cells in vitro. We estimated the proliferation rate of OSCC cells at 0 , 24, 48 and $72 \mathrm{~h}$ after transfection, compared with the negative control, knockdown of HOTAIR notably repressed the growth of OSCC cells (Fig. 2C and D). Furthermore, colony formation assay indicated that the silence of HOTAIR expression significantly reduced colonies of the OSCC cells (Fig. 2E and F).

To further assess the role of HOTAIR in apoptosis and cell death of OSCC cells, we analyzed the HOTAIR knocked down cells using flow cytometry. In addition, the results indicated that knockdown of HOTAIR promoted both early apoptosis and later apoptosis of OSCC cells Tca8113and TSCCA (Fig. 2G and $\mathrm{H}$ ).

We use wound healing scratch assay and Matrigel invasion assay to evaluate whether HOTAIR alters invasion and migra- tion of OSCC cells. As shown, silence of HOTAIR decreased their migration and invasion ability. Conversely, the 2 OSCC cell lines Tca8113 and TSCCA treated with siCT did not affect the cell invasion and migration. Based on our results, expression of HOTAIR promoted migration and invasion of OSCC cells in vitro (Fig. 3).

HOTAIR negatively correlates to E-cadherin expression in OSCC tissues and cell lines. To define functional correlation between HOTAIR and epithelial-mesenchymal transition (EMT), we examined the effects of HOTAIR knockdown on E-cadherin expression. We first assayed the expression level of HOTAIR and E-cadherin in OSCC tissues. A significant negative correlation is observed between the E-cadherin mRNA levels and the HOTAIR expression levels in OSCC tissues $\left(\mathrm{r}^{2}=-0.327, \mathrm{P}=0.004\right)$ (Fig. 4A). Then we investigated whether HOTAIR regulates E-cadherin expression in OSCC cells. Silence of HOTAIR significantly increased E-cadherin expression at both transcript and protein levels in TSCCA and Tca8113 cells (Fig. 4B and C). These results demonstrated that HOTAIR promotes EMT in OSCC tissues and cell lines.

HOTAIR represses E-cadherin expression by associating with EZH2. HOTAIR is thought to regulate transcription by directing the action of PRC2 complexes to control the epigenetic state of the cell and subsequently regulate gene expression. 
A

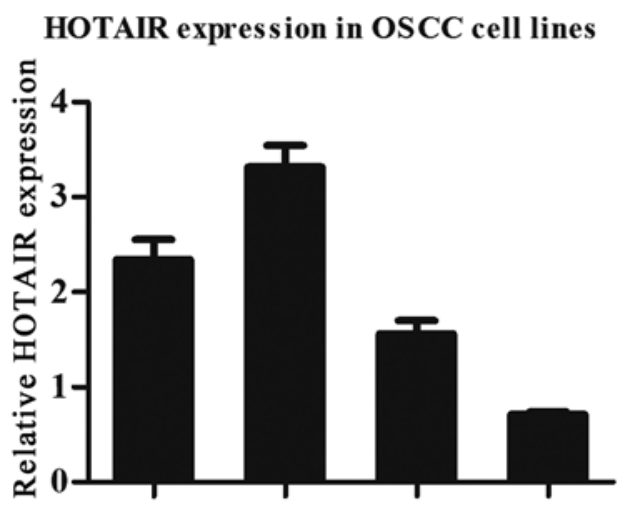

C

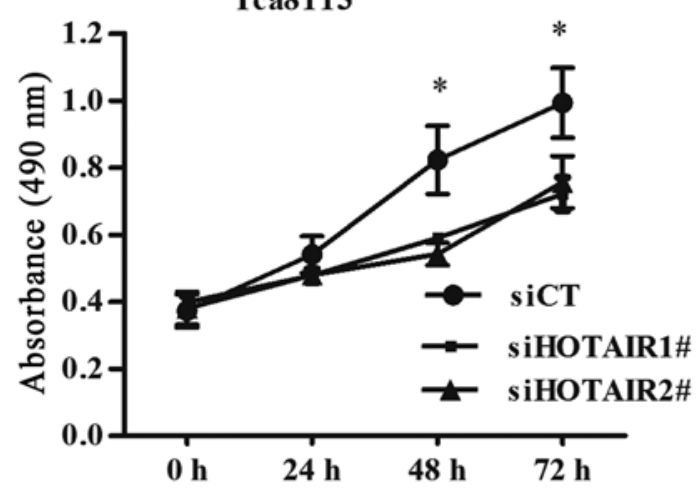

E

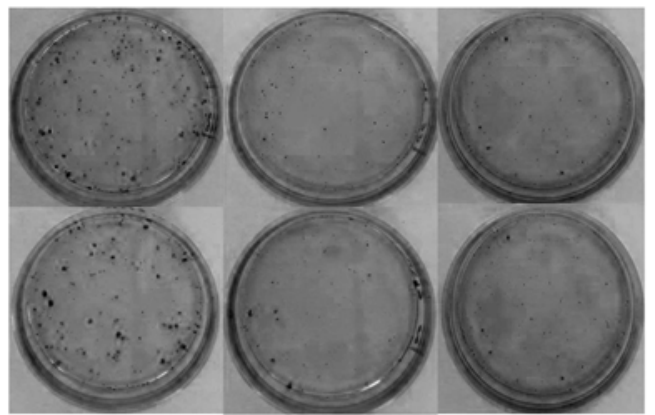

\section{G}
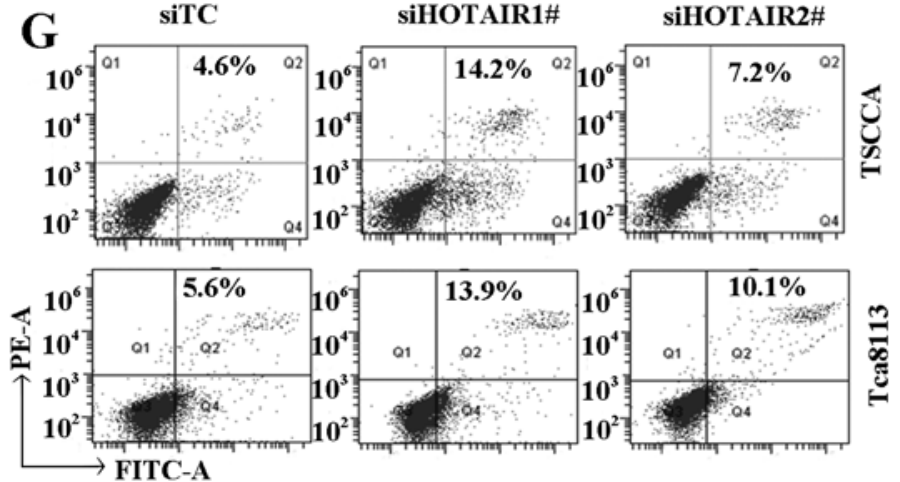

B

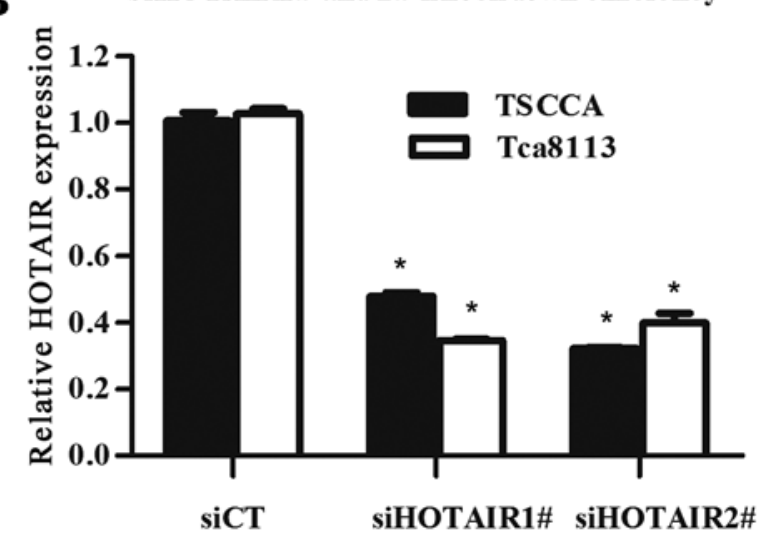

D

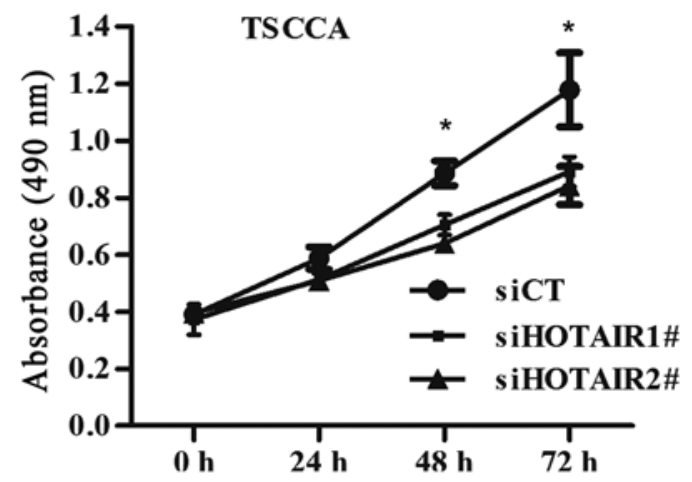

$\mathbf{F}$

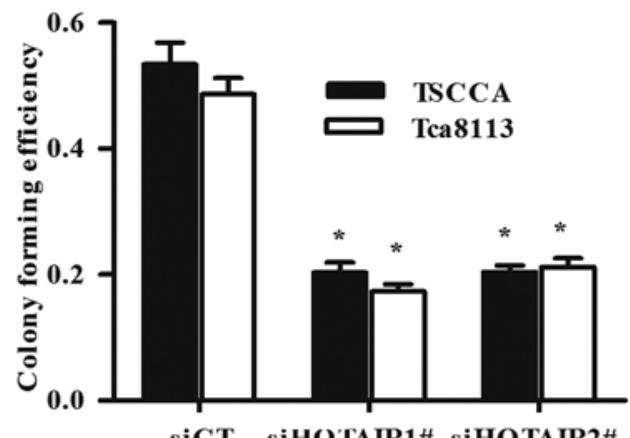

H

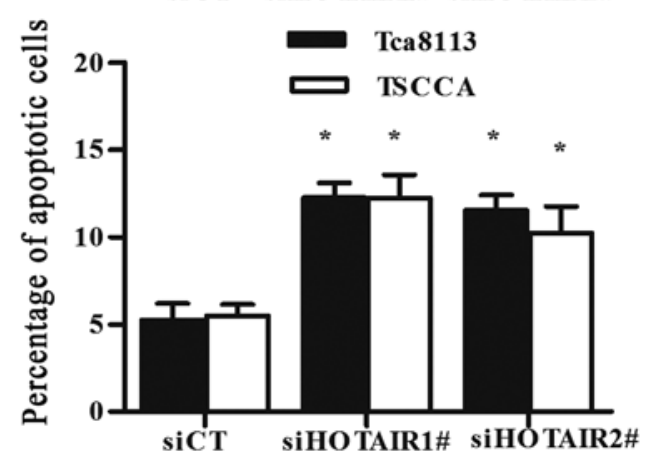

Figure 2. HOTAIR knockdown inhibits the malignant properties of OSCC cells. The expression of HOTAIR in OSCC cell lines as determined by real-time PCR (A) and HOTAIR is efficiently knocked down by siRNA (B) in OSCC cell lines TSCCA and Tca8113. Silencing of HOTAIR suppressed the proliferation of TSCCA and Tca8113 cells as determined by an MTT assay (C and D). The effect of HOTAIR on tumorigenesis of OSCC cells was examined by a colony formation assay, HOTAIR knockdown significantly reduced the anchorage-independent cell growth of TSCCA and Tca8113 cells compared with the negative control (E and F). Annexin V-fluorescein isothiocyanate (FITC)/PI staining of cells transfected with siCT and siHOTAIR for $48 \mathrm{~h}$. The percentage of apoptotic cells were counted and the results are summarized in a bar graph $(\mathrm{G}$ and $\mathrm{H}) .{ }^{*} \mathrm{P}<0.05$.

We performed western blotting to test the enrichment of EZH2 and $\mathrm{H} 3 \mathrm{~K} 27 \mathrm{me} 3$, and expression of E-cadherin in OSCC cell lines treated with HOTAIR siRNA. We observed enrichment of EZH2 and H3K27me3 significantly decreased and with the expression of E-cadherin significantly increased in the HOTAIR knockdown OSCC cells (Fig. 5A). 

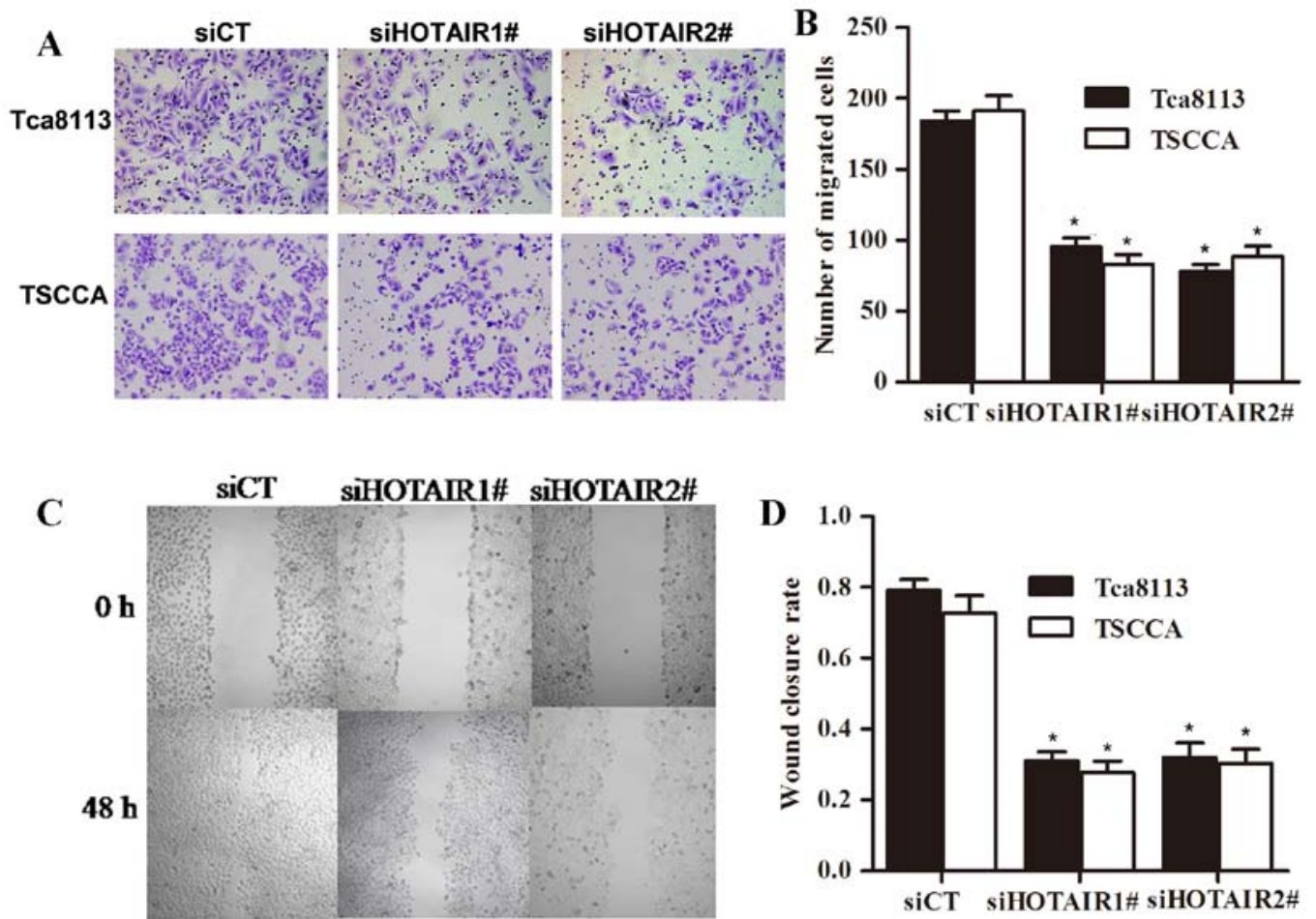

Figure 3. HOTAIR promotes OSCC cell invasion and migration. Wounds were introduced by scratching confluent monolayers of TSCCA and Tca8113 cells transfected with siCT, siHOTAIR. Migration was monitored by light microscopy at 0 and $48 \mathrm{~h}$ (A and B). Matrigel-based invasion assay was performed using modified Boyden chambers with 10\% FBS as a chemoattractant. Representative images are presented. The cell numbers per field were counted and the results are summarized in a bar graph $(\mathrm{C}$ and $\mathrm{D}) .{ }^{*} \mathrm{P}<0.05$.
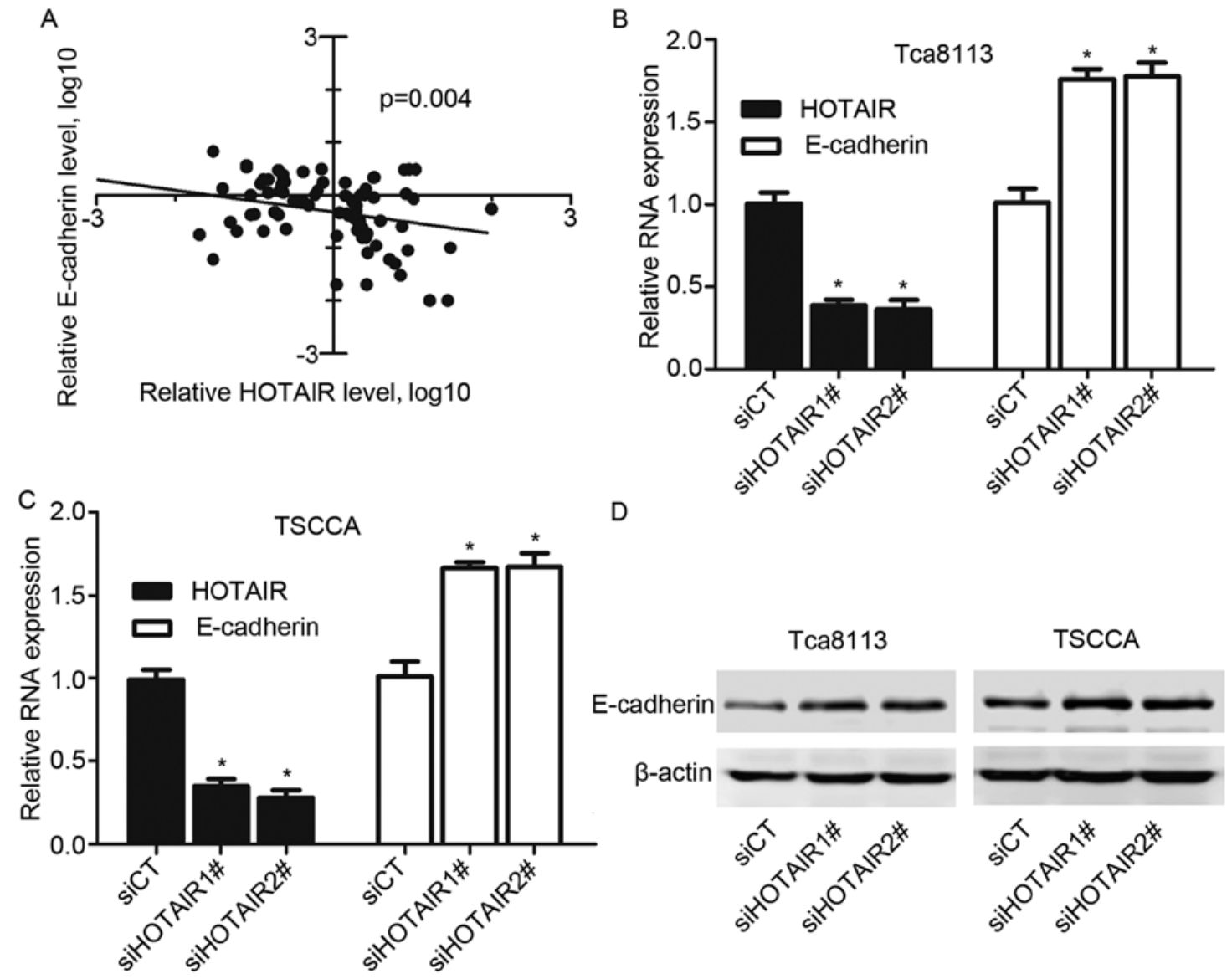

Figure 4. HOTAIR negatively correlates to E-cadherin expression in OSCC tissues and cell lines. Negative correlation between E-cadherin mRNA levels and the HOTAIR levels in 76 OSCC samples (A). Relative expression level of HOTAIR and E-cadherin in Tca813 and TACCA cells treated with siRNA for $48 \mathrm{~h}$ (B and C). Western blot analysis of E-cadherin after siHOTAIR treatment for $48 \mathrm{~h}$ in Tca813 and TACCA cells (D). ${ }^{*} \mathrm{P}<0.05$. 


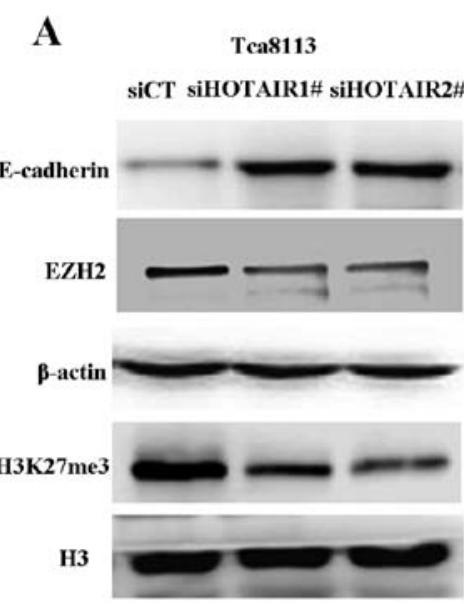

TSCCA
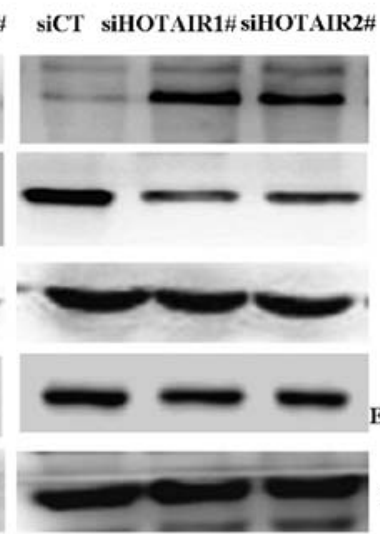

B

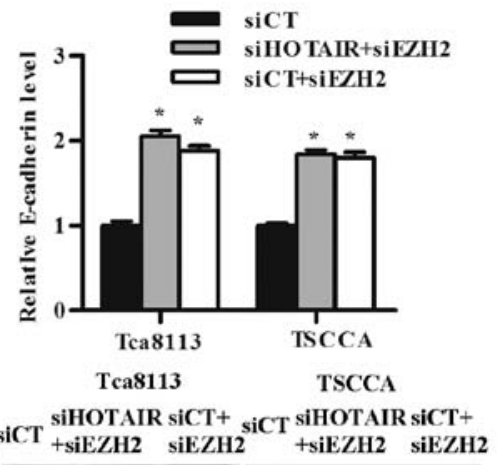

Figure 5. HOTAIR is associated with EZH2 and regulates E-cadherin. Enrichment of EZH2 and H3K27me3 significantly decreased and the expression of E-cadherin significantly increased in the HOTAIR knockdown OSCC cells (A). Relative E-cadherin mRNA and protein level after siHOTAIR and/or siEZH2 treatment in OSCC cells $(\mathrm{B}$ and $\mathrm{C}) .{ }^{*} \mathrm{P}<0.05$.

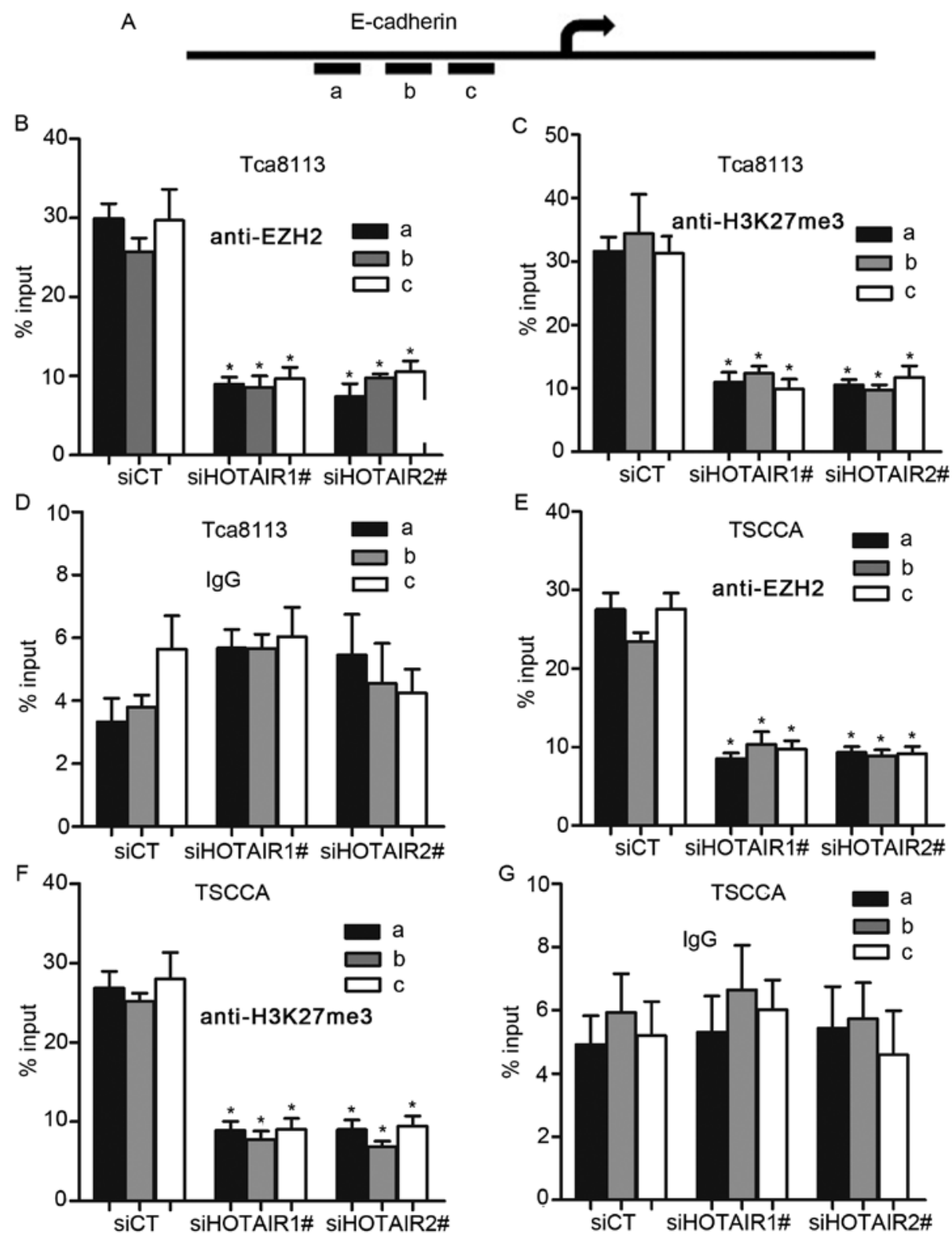

Figure 6. HOTAIR represses E-cadherin expression by associating with EZH2. ChIP analysis of Tca8113 and TSCCA cells treated with siHOTAIR were conducted on E-cadherin promoter (A) using the anti-EZH2 and anti-H3K27me3 antibodies and IgG. Enrichment was determined relative to input controls (B-G). ${ }^{*} \mathrm{P}<0.05$. 
To check whether expression of the E-cadherin was controlled by EZH2, we analyzed the E-cadherin expression after EZH2 and/or HOTAIR knockdown. E-cadherin mRNA and protein levels increased in Tca8113 and TSCCA cells treated with siEZH2 and siEZH2+siHOTAIR groups (Fig. 5B and $\mathrm{C}$ ).

To further address how HOTAIR regulated E-cadherin through enrichment of EZH2, we performed ChIP-arrays in OSCC cell lines with HOTAIR knockdown. In addition, results showed that HOTAIR silence decreased the binding of EZH2 and $\mathrm{H} 3 \mathrm{~K} 27 \mathrm{me} 3$ with the E-cadherin promoter in OSCC cells, whereas, we did not detect the binding of $\mathrm{IgG}$ with E-cadherin promoter after HOTAIR inhibition (Fig. 6B-G). These results suggest that HOTAIR represses E-cadherin expression partly by associating with EZH2.

\section{Discussion}

Evidence has shown that HOTAIR can act as a key regulation factor in the molecular mechanisms underlying the development and progression of cancer $(12,13)$, therefore potentially leading to new strategies for cancer therapy. In addition, in the clinic, it is necessary to solve the problems to improve the treatment outcome in patients of OSCC, therefore exploring new strategies for molecular targeted therapy is urgent needed. As a novel lncRNA, HOTAIR initially become well-known for its involvement in cancer invasiveness and metastasis $(10,14-17)$, and is expected to be the most potential treatment target in OSCC.

Several reports have suggested that HOTAIR is upregulated in breast cancer, colorectal cancer, esophageal squamous cell carcinoma (ESCC) and promotes cancer invasion and metastasis and poor survival $(10,14,16-21)$. However, this study is the first to demonstrate the regulation of HOTAIR in OSCC and revealed that HOTAIR expression is correlated with the aggressive biological behavior of OSCC. In the present study, HOTAIR is overexpressed in OSCC tissues and cell lines. In addition, the patients with high HOTAIR expression had more cervical lymph node metastasis, more advanced stage and poorer histological differentiation than those with low HOTAIR expression. However, other clinicopathological factors, such as tumor size, age, gender and smoking and/or alcohol abuse, were not correlated with the expression of HOTAIR. In addition, it is known that patients with oral tongue squamous cell carcinoma (OTSCC) have a significantly worse prognosis than those with similar lesions of the other oral cavity sites (22), however, the expression of HOTAIR did not show a significant difference between the two groups. Besides, we found that the patients with high level of HOTAIR had lower OS rate and DFS rate which suggested that overexpression of HOTAIR might be an indicator of poor outcome in OSCC patients.

Emerging evidence suggests that HOTAIR regulates proliferation, invasion and apoptosis of a variety of tumor cells in vitro $(15,18,23,24)$. Enforced expression of HOTAIR in breast carcinoma cells promoted cell invasion (10), knockdown of HOTAIR reduced pancreatic cancer cell invasion, inhibited cell growth, modulated cell cycle progression and induced apoptosis (14), and the results were confirmed in colorectal cancer (16), non-small cell lung cancer $(17,25,26)$, gastric cancer (27-29) and other cancers (15,20,30-32). To further understand the biological function of HOTAIR in OSCC progression, in vitro assays were performed in the OSCC cell lines TSCCA and Tca8113. Our data showed that knockdown of HOTAIR mediated by siRNA led to significant decrease in proliferation, colony forming efficiency and invasive ability and significant increase in apoptosis in OSCC cells.

Determination of the targeted proteins associated with HOTAIR and the genes regulated by HOTAIR would reveal the molecular mechanisms underlying cancer development and progression by HOTAIR. HOTAIR functions in the recruitment and binding of the PRC2 and LSD1 complexes to the HOXD locus on chromosome 2 where genes involved in metastasis are regulated through $\mathrm{H} 3 \mathrm{~K} 27$ methylation and $\mathrm{H} 3 \mathrm{~K} 4$ demethylation $(9,33)$. Reports have revealed that HOTAIR led to decreased expression of some mesenchymal markers, while the expression of epithelial markers (such as E-cadherin) were increased in various cancers $(20,34,35)$. EZH2, a unit of the PRC2 complex, has been reported to downregulate the level of E-cadherin gene expression through $\mathrm{H} 3 \mathrm{~K} 27 \mathrm{me} 3$ in the E-cadherin promoter (36-38). Thus, we speculate HOTAIR induces EMT related markers through EZH2. In our study, we found a significantly negative correlation between HOTAIR levels and E-cadherin levels in OSCC tissues and cell lines. In addition, knocking down of $\mathrm{EZH} 2$, but not changing the HOTAIR expression level could also upregulate E-cadherin expression in vivo. Furthermore, we performed ChIP analysis in HOTAIR-silenced OSCC cell lines, and results showed that HOTAIR contributes to the binding of EZH2 and H3K27me3 with the E-cadherin promoter.

In conclusion, this study has shown that HOTAIR, defined as an oncogene is overexpressed in OSCC patients and indicates poor prognosis, and poor outcome of patients with high HOTAIR expression due to the ability of HOTAIR to promote cancer development and progression by inducing cancer cell proliferation and invasion and reducing cancer cell apoptosis. Moreover, HOTAIR regulates EMT related marker E-cadherin through recruitment of EZH2 and H3K27me3. Therefore, these findings indicate that HOTAIR plays a vital role in the development and progression of OSCC and downregulation of such lncRNAs could be a valuable predictive marker as well as novel therapeutic target in future OSCC treatment.

\section{Acknowledgements}

This study was supported by National Basic Research Program of China (973 Program), no. 2010CB529301 (to Xishan Hao) and supported in part by the China National Natural Scientific Fund (81172573) (to Lun Zhang).

\section{References}

1. Siegel R, Ma J, Zou Z and Jemal A: Cancer statistics, 2014. CA Cancer J Clin 64: 9-29, 2014.

2. Molinolo AA, Amornphimoltham P, Squarize CH, Castilho RM, Patel V and Gutkind JS: Dysregulated molecular networks in head and neck carcinogenesis. Oral Oncol 45: 324-334, 2009.

3. Sabour S and Moezizadeh M: Prediction of OSCC using biomarkers: Methodological mistake. Oral Oncol 48: e51, 2012.

4. Raj LSM, Boaz K and Natarajan S: Prognostic significance of lymph node pattern in oral squamous cell carcinoma (OSCC). J Clin Diagn Res 8: 232-235, 2014. 
5. Grimm M: Prognostic value of clinicopathological parameters and outcome in 484 patients with oral squamous cell carcinoma: Microvascular invasion ( $\mathrm{V}+$ ) is an independent prognostic factor for OSCC. Clin Transl Oncol 14: 870-880, 2012.

6. Wang KC and Chang HY: Molecular mechanisms of long noncoding RNAs. Mol Cell 43: 904-914, 2011.

7. Lee JT: Epigenetic regulation by long noncoding RNAs. Science 338: 1435-1439, 2012.

8. Kung JT, Colognori D and Lee JT: Long noncoding RNAs: Past, present, and future. Genetics 193: 651-669, 2013.

9. Tsai MC, Manor O, Wan Y, Mosammaparast N, Wang JK, Lan F, Shi Y, Segal E and Chang HY: Long noncoding RNA as modular scaffold of histone modification complexes. Science 329: 689-693, 2010

10. Gupta RA, Shah N, Wang KC, Kim J, Horlings HM, Wong DJ, Tsai MC, Hung T, Argani P, Rinn JL, et al: Long non-coding RNA HOTAIR reprograms chromatin state to promote cancer metastasis. Nature 464: 1071-1076, 2010.

11. Rinn JL, Kertesz M, Wang JK, Squazzo SL, Xu X, Brugmann SA, Goodnough LH, Helms JA, Farnham PJ, Segal E, et al: Functional demarcation of active and silent chromatin domains in human HOX loci by noncoding RNAs. Cell 129: 1311-1323, 2007.

12. Woo CJ and Kingston RE: HOTAIR lifts noncoding RNAs to new levels. Cell 129: 1257-1259, 2007.

13. Sun L and Fang J: Writer meets eraser in HOTAIR. Acta Biochim Biophys Sin (Shanghai) 43: 1-3, 2011.

14. Kim K, Jutooru I, Chadalapaka G, Johnson G, Frank J, Burghardt R, Kim S and Safe S: HOTAIR is a negative prognostic factor and exhibits pro-oncogenic activity in pancreatic cancer. Oncogene 32: 1616-1625, 2013.

15. Tang L, Zhang W, Su B and Yu B: Long noncoding RNA HOTAIR is associated with motility, invasion, and metastatic potential of metastatic melanoma. BioMed Res Int 2013: 251098, 2013.

16. Kogo R, Shimamura T, Mimori K, Kawahara K, Imoto S, Sudo T, Tanaka F, Shibata K, Suzuki A, Komune S, et al: Long noncoding RNA HOTAIR regulates polycomb-dependent chromatin modification and is associated with poor prognosis in colorectal cancers. Cancer Res 71: 6320-6326, 2011.

17. Liu XH, Liu ZL, Sun M, Liu J, Wang ZX and De W: The long non-coding RNA HOTAIR indicates a poor prognosis and promotes metastasis in non-small cell lung cancer. BMC Cancer 13: 464, 2013.

18. Ono $\mathrm{H}$, Motoi $\mathrm{N}$, Nagano $\mathrm{H}$, Miyauchi E, Ushijima $\mathrm{M}$ Matsuura M, Okumura S, Nishio M, Hirose T, Inase N, et al: Long noncoding RNA HOTAIR is relevant to cellular proliferation, invasiveness, and clinical relapse in small-cell lung cancer. Cancer Med 3: 632-642, 2014.

19. Li X, Wu Z, Mei Q, Li X, Guo M, Fu X and Han W: Long noncoding RNA HOTAIR, a driver of malignancy, predicts negative prognosis and exhibits oncogenic activity in oesophageal squamous cell carcinoma. Br J Cancer 109: 2266-2278, 2013

20. Qiu JJ, Lin YY, Ye LC, Ding JX, Feng WW, Jin HY, Zhang Y, Li Q and Hua KQ: Overexpression of long non-coding RNA HOTAIR predicts poor patient prognosis and promotes tumor metastasis in epithelial ovarian cancer. Gynecol Oncol 134: 121-128, 2014

21. Chen FJ, Sun M, Li SQ, Wu QQ, Ji L, Liu ZL, Zhou GZ, Cao G, Jin L, Xie HW, et al: Upregulation of the long non-coding RNA HOTAIR promotes esophageal squamous cell carcinoma metastasis and poor prognosis. Mol Carcinog 52: 908-915, 2013.

22. Bello IO, Soini Y and Salo T: Prognostic evaluation of oral tongue cancer: Means, markers and perspectives (I). Oral Oncol 46: $630-635,2010$

23. Bhan A, Hussain I, Ansari KI, Bobzean SA, Perrotti LI and Mandal SS: Bisphenol-A and diethylstilbestrol exposure induces the expression of breast cancer associated long noncoding RNA HOTAIR in vitro and in vivo. J Steroid Biochem Mol Biol 141: 160-170, 2014.
24. He X, Bao W, Li X, Chen Z, Che Q, Wang H and Wan XP: The long non-coding RNA HOTAIR is upregulated in endometrial carcinoma and correlates with poor prognosis. Int J Mol Med 33: 325-332, 2014

25. Zhuang Y, Wang X, Nguyen HT, Zhuo Y, Cui X, Fewell C, Flemington EK and Shan B: Induction of long intergenic noncoding RNA HOTAIR in lung cancer cells by type I collagen. J Hematol Oncol 6: 35, 2013

26. Nakagawa T, Endo H, Yokoyama M, Abe J, Tamai K, Tanaka N, Sato I, Takahashi S, Kondo T and Satoh K: Large noncoding RNA HOTAIR enhances aggressive biological behavior and is associated with short disease-free survival in human non-small cell lung cancer. Biochem Biophys Res Commun 436: 319-324, 2013.

27. Endo H, Shiroki T, Nakagawa T, Yokoyama M, Tamai K, Yamanami H, Fujiya T, Sato I, Yamaguchi K, Tanaka N, et al: Enhanced expression of long non-coding RNA HOTAIR is associated with the development of gastric cancer. PLoS One 8: e77070, 2013

28. Xu ZY, Yu QM, Du YA, Yang LT, Dong RZ, Huang L, Yu PF and Cheng XD: Knockdown of long non-coding RNA HOTAIR suppresses tumor invasion and reverses epithelial-mesenchymal transition in gastric cancer. Int J Biol Sci 9: 587-597, 2013.

29. Hajjari M, Behmanesh M, Sadeghizadeh M and Zeinoddini M: Up-regulation of HOTAIR long non-coding RNA in human gastric adenocarcinoma tissues. Med Oncol 30: 670, 2013.

30. Ge XS, Ma HJ, Zheng XH, Ruan HL, Liao XY, Xue WQ, Chen YB, Zhang Y and Jia WH: HOTAIR, a prognostic factor in esophageal squamous cell carcinoma, inhibits WIF-1 expression and activates Wnt pathway. Cancer Sci 104: 1675-1682, 2013.

31. Li D, Feng J, Wu T, Wang Y, Sun Y, Ren J and Liu M: Long intergenic noncoding RNA HOTAIR is overexpressed and regulates PTEN methylation in laryngeal squamous cell carcinoma. Am J Pathol 182: 64-70, 2013

32. Niinuma T, Suzuki H, Nojima M, Nosho K, Yamamoto $H$, Takamaru H, Yamamoto E, Maruyama R, Nobuoka T, Miyazaki Y, et al: Upregulation of miR-196a and HOTAIR drive malignant character in gastrointestinal stromal tumors. Cancer Res 72: 1126-1136, 2012.

33. Wu L, Murat P, Matak-Vinkovic D, Murrell A and Balasubramanian S: Binding interactions between long noncoding RNA HOTAIR and PRC2 proteins. Biochemistry 52: 9519-9527, 2013

34. Zhang H, Cai K, Wang J, Wang X, Cheng K, Shi F, Jiang L, Zhang Y and Dou J: MiR-7, inhibited indirectly by lincRNA HOTAIR, directly inhibits SETDB1 and reverses the EMT of breast cancer stem cells by downregulating the STAT3 pathway. Stem Cells 32: 2858-2868, 2014

35. Pádua Alves C, Fonseca AS, Muys BR, de Barros E, Lima Bueno R, Bürger MC, de Souza JE, Valente V, Zago MA and Silva WA Jr: Brief report: The lincRNA Hotair is required for epithelial-to-mesenchymal transition and stemness maintenance of cancer cell lines. Stem Cells 31: 2827-2832, 2013.

36. Wang C, Liu X, Chen Z, Huang H, Jin Y, Kolokythas A, Wang A, Dai Y, Wong DT and Zhou X: Polycomb group protein EZH2mediated E-cadherin repression promotes metastasis of oral tongue squamous cell carcinoma. Mol Carcinog 52: 229-236, 2013.

37. Liu L, Xu Z, Zhong L, Wang H, Jiang S, Long Q, Xu J and Guo J: EZH2 promotes tumor cell migration and invasion via epigenetic repression of E-cadherin in renal cell carcinoma. BJU Int: Feb 25, 2014 (Epub ahead of print). doi: 10.1111/bju.12702.

38. Sun NX, Ye C, Zhao Q, Zhang Q, Xu C, Wang SB, Jin ZJ, Sun SH, Wang $\mathrm{F}$ and $\mathrm{Li} \mathrm{W}$ : Long noncoding RNA-EBIC promotes tumor cell invasion by binding to EZH2 and repressing E-cadherin in cervical cancer. PLoS One 9: e100340, 2014 